\author{
Military Technical College \\ Kobry Elkobbah, \\ Cairo, Egypt
}

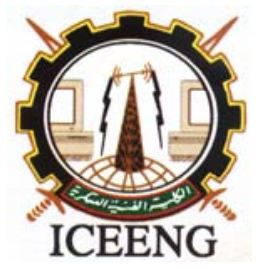

\author{
$5^{\text {th }}$ International Conference \\ on Electrical Engineering \\ ICEENG 2006
}

\title{
DIRECTION OF ARRIVAL ESTIMATION VIA EXPLOITATION OF CYCLOSTATIONARITY IN A SPACE / TIME ARRAY PROCESSING ALGORITHM
}

\author{
ELSAYED E. AZZOUZ * \\ ASHRAF M. ABD EL-AZIZ ** \\ M. A. Abo El-Azm **
}

\begin{abstract}
:
This paper is concerned with introducing a direction of arrival estimation (DOA) estimation algorithm that is independent of the bandwidth of the intercepted signals. The proposed algorithm offers a modification to most subspace based DOA estimation algorithms that depend basically on narrowband signals assumption. The basic idea of this modification is the replacement of conventional correlation matrix by a cyclic one. This modification enables the proposed algorithm to deal with both narrowband and wideband signals. In addition, this algorithm requires only that the interference signals are cyclically uncorrelated with SOI. This condition is easier to be achieved than uncorrelation condition that is required by conventional algorithms. Computer simulations using an additive white Gaussian noise (AWGN) channel show that the proposed DOA estimation algorithm succeeds to estimate DOAs of the SOI and interference signals at $10 \mathrm{~dB}$ signal to noise ratio and $-3 \mathrm{~dB}$ signal to interference ratio.
\end{abstract}

\section{I-Introduction:}

Generally, the performance of the spatial structure DOA estimation algorithms that depend on knowing spatial antenna array information are limited by the accuracy of that information and 
the number of array elements [1]. Also, temporal structure DOA estimation algorithms that depend on restoring a temporal property of the intercepted signals do not make explicit use of the available knowledge of spatial structure of the antenna array. The proposed DOA estimation algorithm combines spatial structure information of the array and temporal structure information of the intercepted signals. Thus, the proposed algorithm is considered as a spatial temporal process. Multiple signal classification (MUSIC) algorithm is used as a type of subspace based algorithms [1]. Cyclostationarity property based algorithms are used as the temporal part of the proposed algorithm. Therefore, the proposed algorithm is called cyclic MUSIC algorithm. Conventional cyclic subspace algorithms were based on the assumption that all incident signals on the array have narrow bandwidth otherwise their performance degrades as the signals bandwidths increase. However, it will be shown that proposed cyclic MUSIC algorithm is robust for DOA estimation of both narrowband and wideband signal of interest (SOI) or interference signals. If all incident interference signals on the array are mutually cyclically uncorrelated with SOI, the spatial spectrum of cyclic MUSIC algorithm produces only one peak that corresponds to SOI direction. However, in presence of mutually cyclically correlated signals, the proposed algorithm can determine only DOAs of the SOI and its cyclically correlated signals.

This paper is organized as follows: Section II presents a simple uniform linear array (ULA) data model for analysis study. At first, a short description of the basic concepts of cyclostatioarity process is introduced. Then, a more general data model is presented, followed by introducing the proposed algorithm for DOA estimation. To understand the difference between the existing cyclic algorithms and the proposed one, a brief description of the existing algorithms is presented and compared with the proposed cyclic MUSIC algorithm. The simulation results and discussions are given in section III. Finally, the conclusions are provided in section IV.

\section{II- Uniformly Spaced Linear Array Data Model}

\section{A- Basic concepts of cyclostatioarity process}

Cyclostatioarity is a physical phenomenon that describes a probabilistic model for certain random data that involves certain periodicity model. For example, the utilized signal periodicity in communication telemetry, radar, and sonar arises from sampling, scanning, modulation, multiplexing, and coding operations. There is more than one definition of cyclostationarity. First, a signal, ${ }^{\mathbf{x}(t)}$, is said to be cyclostationary in a wide sense if its mean, $m_{x}(t)$, and autocorrelation, $\mathbf{R}_{x}(t, u)$, are periodic with the same period, ${ }^{T}$.

$$
\begin{aligned}
& m_{x}\left(t+T_{p}\right)=m_{x}(t) \\
& \mathbf{R}_{x}\left(t+T_{p}, u+T_{p}\right)=\mathbf{R}_{x}(t, u)
\end{aligned}
$$

for all $t$ and $u$ where $m_{x}(t)=E\{\mathbf{x}(t)\}$ and $\mathbf{R}_{x}(t, u)=E\left\{\mathbf{x}(t) \mathbf{x}^{*}(t+u)\right\}$. The autocorrelation function can be re-expressed as:

$$
\mathbf{R}_{x}(t+\tau / 2, t-\tau / 2) \stackrel{\Delta}{=} E\left\{\mathbf{x}(t+\tau / 2) \mathbf{x}^{*}(t-\tau / 2)\right\}
$$


where $\mathrm{t}$ and $\tau$ are independent time variables. Thus, $\mathbf{R}_{x}(t+\tau / 2, t-\tau / 2)$ is periodic in time with period $T_{p} \neq 0$ for each value of $\tau$. It is assumed that the Fourier series representation for this periodic function converges such that $\mathbf{R}_{x}$ can be expressed as [2]:

$$
\mathbf{R}_{x}(t+\tau / 2, t-\tau / 2)=\sum_{\alpha} \mathbf{R}_{x}^{\alpha}(\tau) e^{j 2 \pi \alpha t}
$$

for which $\left\{\mathbf{R}_{x}^{\alpha}(\tau)\right\}$ are the Fourier series coefficients and they are given by:

$$
\mathbf{R}_{x}^{\alpha}(\tau)=\frac{1}{T_{p}} \int_{\frac{-T_{p}}{2}}^{\frac{T_{p}}{2}} \mathbf{R}_{x}(t+\tau / 2, t-\tau / 2) e^{-j 2 \pi \alpha t} d t
$$

$\mathbf{R}_{x}^{\alpha}(\tau)$ is called cyclic autocorrelation function with cyclic frequency $\alpha$. This cyclic frequency ranges over all integer multiples of the $i^{\text {th }}$ parameter of periodicity, ${ }^{j / T_{i}}$, (such as carrier frequency, baud rate, and their sums and differences ) [2] where $j$ takes an integer value. If conjugate operation is removed from (3), then by substituting in (5), the cyclic correlation is called conjugate cyclic correlation $\mathbf{R}_{x^{*}}^{\alpha}(\tau)$. There are some signals that have both nonzero cyclic and nonzero conjugate cyclic correlations such as BPSK signals $[2,3]$. More generally, a non stationary signal $\mathbf{x}(t)$ is said to exhibit cyclostationarity if there exist a cyclic frequency $\alpha$ for which $\mathbf{R}_{x}^{\alpha}(\tau)$ is not identically zero. Note that, for a stationary $\operatorname{signal}_{x} \mathbf{R}_{x}^{\alpha}(\tau)$ will be zero for all nonzero $\alpha$.

Another definition of cyclostationarity is to let $\mathbf{U}(t)$ and $\mathbf{V}(t)$ be the frequency shift versions of $\mathbf{x}(t)$.

$$
\begin{aligned}
& \mathbf{U}(t) \stackrel{\Delta}{=} \mathbf{x}(t) e^{-j 2 \pi \alpha t} \\
& \mathbf{V}(t) \stackrel{\Delta}{=} \mathbf{x}(t) e^{+j 2 \pi \alpha t}
\end{aligned}
$$

The time averaged cross correlation can be defined as $[2,3]$ :

$$
<\mathbf{R}_{U V}>(\tau) \stackrel{\Delta}{=} \lim _{T_{p} \rightarrow \infty} \frac{1}{T_{p}} \int_{\frac{-T_{p}}{2}}^{\frac{T_{p}}{2}} \mathbf{U}(t+\tau / 2) \mathbf{V}^{*}(t-\tau / 2) d t \equiv \mathbf{R}_{x}^{\alpha}(\tau)
$$

where $<\mathbf{R}>$ denotes for the time average of $\mathbf{R}$. Therefore, the cyclic autocorrelation of the signal $\mathbf{x}(t)$ is simply the time average cross correlation between the two frequency shift versions of this signal ${ }^{\mathbf{x}}(t)$. This reveals that a signal exhibits cyclostationarity in wide sense only if there exists correlation between some frequency shift versions of that signal. Thus, signals that use either a different carrier frequencies or different symbol rates will have different spectral correlation functions even though they might occupy the same bandwidth. Assuming that several signals with different spectral correlation functions are impinging on an antenna array, it is possible to construct a linear combiner that nulls out the unwanted signals by using only the selectivity contained in the cyclic frequencies $[4,5]$. 
One major drawback of using only the temporal cyclic approach for DOA estimation is the fact that different symbol rates and / or different carrier frequencies are needed for separating multiple superimposed signals. However, in most communication systems, all users have the same symbol rates. Thus, it is desired to exploit the spatial information in order to separate between those superimposed signals. Subsection B introduces the data model of the intercepted signal by a ULA array. Subsection C presents a brief description of conventional cyclic subspace algorithms that exploit cyclostationarity property for DOA estimation. Subsection D presents the proposed cyclic subspace algorithm, which is independent on the bandwidth of the intercepted signals.

\section{B- Data model}

Consider an $M$-elements ULA array, which are equally spaced by a distance $d$. Moreover, there are a (known / estimated) number of signals $s_{1}(t), \ldots \ldots \ldots \ldots, s_{q}(t) \quad[1,6]$ all centered on a known frequency, $f_{c}$. These signals are impinging on the M-elements antenna array with DOAs $\phi_{i}, i=1,2, \ldots . ., q$. Thus, any intercepted signal by the $i^{\text {th }}$ element of the array is a superposition of the complex envelope of all the impinging signals, $\widetilde{s}_{k}(t) \forall k=1,2, \ldots \ldots, q$, and a noise signal, $\widetilde{n}_{i}(t)$,of variance $\sigma_{n}^{2}$ and has (AWGN) distribution. Therefore, the intercepted signal on the $i^{\text {th }}$ element of the array is given by:

$$
x_{i}(t)=\sum_{k=1}^{q} \widetilde{s}_{k}\left(t+\frac{(i-1)}{c} d \sin \phi_{k}\right)+\widetilde{n}_{i}(t) \forall i=1,2, \ldots . ., M
$$

If all the $s_{i}(t)$ are narrowband with the same center frequency, $f_{c}$, then (9) can b approximated as [1]:

$$
\mathbf{x}(t) \approx \sum_{i=1}^{q} \mathbf{a}\left(\phi_{i}\right) \widetilde{s}_{i}(t)+\mathbf{n}(t)
$$

where $\mathbf{a}\left(f_{c}, \phi_{i}\right)$ is the steering vector of the ith incident signal with DOA $\phi_{i}$

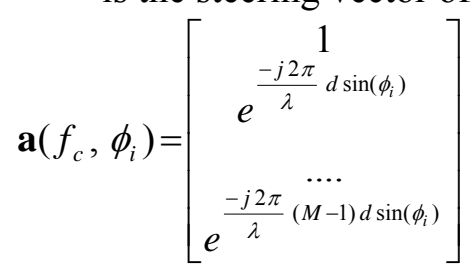

In matrix notation, (10) becomes

$$
\mathbf{x}(t)=\mathbf{A}\left(f_{c}, \phi\right) \mathbf{s}(t)+\mathbf{n}(t)
$$

where $\mathbf{A}\left(f_{c}, \phi\right)$ is the $\mathrm{Mx}$ q matrix of the steering vectors

$$
\mathbf{A}\left(f_{c}, \phi\right)=\left[\mathbf{a}\left(\phi_{1}\right), \ldots \ldots \ldots \ldots \ldots \ldots, \mathbf{a}\left(\phi_{q}\right)\right]^{T}
$$

$\mathbf{n}(t)$ is the complex envelope vector of an AWGN signals along the array elements, 


$$
\mathbf{n}(t)=\left[\widetilde{n}_{1}(t), \ldots \ldots \ldots \ldots, \widetilde{n}_{M}(t)\right]^{T}
$$

and $\mathbf{s}(t)$ is the complex envelope vector of the incident signals

$$
\mathbf{s}(t)=\left[\widetilde{s}_{1}(t), \ldots \ldots \ldots \ldots . . ., \widetilde{s}_{q}(t)\right]^{T}
$$

It is noted that (12) represents the most commonly used narrowband input data model.

\section{C - Conventional cyclic subspace algorithms}

In this subsection, a brief summery of the cyclic subspace algorithms for DOA estimation is presented. The cyclic subspace algorithms use the following assumptions:

A1: $q$ source signals are mutually cyclically uncorrelated. This means that cyclic cross correlation of any pair of source signals is zero at each cyclic frequency $\alpha$ of interest. i.e. $R_{s_{i} s_{j}}^{\alpha}(\tau)=0 \forall i, j \in\{1,2, \ldots ., q\} ; i \neq j$. Note that, this condition is weaker than requiring mutually uncorrelated sources because uncorrelated signals are always cyclically uncorrelated while cyclically uncorrelated signals can be mutually correlated $[3,4]$.

A2: There exist $d_{\alpha} \leq q$ source signals sharing the same cyclic frequency $\alpha$, where $\alpha$ is known or estimated [5]. Thus, there exist only $d_{\alpha}$ mutually cyclically correlated signals have cyclic frequency $\alpha$ i.e. $R_{s_{i} s_{i}}^{\alpha}(\tau) \neq$ zero $\forall i \in\left\{1,2, \ldots . ., d_{\alpha}\right\}$.

A3: It is only required that noise source signals are not be cyclically correlated with themselves and with other source signals at the cyclic frequency of interest $\alpha$. This condition is weaker than having jointly stationary noise source signals. From equations (6) and (7), since

$$
U(t+\tau / 2) V^{*}(t-\tau / 2) \equiv \mathbf{X}(t+\tau / 2) \mathbf{X}^{H}(t-\tau / 2) e^{-j 2 \pi \alpha t}
$$

Then by substituting equations (12) and (16) into equation (8), the cross cyclic correlation is estimated as:

$$
\mathbf{R}_{x}^{\alpha}(\tau)=<\left[\mathbf{A}\left(f_{o}\right) \mathbf{S}(t+\tau / 2)+\mathbf{n}(t+\tau / 2)\right]\left[\mathbf{A}\left(f_{o}\right) \mathbf{S}(t-\tau / 2)+\mathbf{n}(t-\tau / 2)\right]^{H} e^{-j 2 \pi \alpha t}>
$$

From Using assumptions A1, A2, and A3, the cross cyclic correlation can be re-expressed as:

$$
\mathbf{R}_{S}^{\alpha}(\tau)=<\mathbf{S}(t+\tau / 2) \mathbf{S}^{H}(t-\tau / 2) e^{-j 2 \pi \alpha t}>
$$

where

$$
\mathbf{R}_{x}^{\alpha}(\tau)=\mathbf{A}\left(f_{o}\right) \mathbf{R}_{S}^{\alpha}(\tau) \mathbf{A}^{H}\left(f_{o}\right)
$$

The basic idea of the cyclic subspace algorithms is the replacement of conventional

correlation matrix, defined in equation (3), by the cyclic correlation matrix, $\mathbf{R}_{x}^{\alpha}(\tau)$, in attempt to null out or reduce interference from the output of the array.

\section{D - Upgraded cyclic subspace algorithm}

Starting from the general data model for the array output signal, cyclic correlation of the intercepted signal by each sensor is given by: 


$$
\begin{aligned}
R_{x_{i}}^{\alpha}(\tau) & =<x_{i}(t+\tau / 2) x_{i}^{H}(t-\tau / 2) e^{-j 2 \pi \alpha t}> \\
& =<\left[\sum_{k=1}^{q} \widetilde{s}_{k}\left(t+\frac{(i-1)}{c} d \sin \theta_{k}\right)+\widetilde{n}_{i}(t)\right]\left[\sum_{k=1}^{q} \widetilde{s}_{k}^{*}\left(t+\frac{(i-1)}{c} d \sin \theta_{k}\right)+\widetilde{n}_{i}^{*}(t)\right] e^{-j 2 \pi \alpha t}>
\end{aligned}
$$

From A1, A2, and A3, the double summation is reduced into a single sum. Thus, ${ }^{x_{x_{i}}}(\tau)$ can be written as:

$$
R_{x_{i}}^{\alpha}(\tau)=\sum_{k=1}^{d_{\alpha}}<\widetilde{s}_{k}\left(t+\frac{(i-1)}{c} d \sin \theta_{k}+\frac{\tau}{2}\right) \widetilde{s}_{k}^{*}\left(t+\frac{(i-1)}{c} d \sin \theta_{k}-\frac{\tau}{2}\right) e^{-j 2 \pi \alpha t}>
$$

As proved in appendix (A), the temporal delay in a signal reveals itself as a phase shift on the cyclic correlation of that signal. Consequently,

$$
\begin{aligned}
<\widetilde{S}_{k}\left(t+\frac{(i-1)}{c} d \sin \theta_{k}+\frac{\tau}{2}\right) \widetilde{s}_{k}^{*}\left(t+\frac{(i-1)}{c} d \sin \theta_{k}-\frac{\tau}{2}\right) e^{-j 2 \pi \alpha t}> \\
=<\widetilde{s}_{k}\left(t+\frac{\tau}{2}\right) \widetilde{s}_{k}^{*}\left(t-\frac{\tau}{2}\right) e^{-j 2 \pi \alpha t}>e^{j 2 \pi f_{o} \frac{(i-1)}{c} d \sin \theta_{k}} \\
=R_{s_{k}}^{\alpha}(\tau) e^{j 2 \pi f_{o} \frac{(i-1)}{c} d \sin \theta_{k}}
\end{aligned}
$$

and

$$
R_{x_{i}}^{\alpha}(\tau)=\sum_{k=1}^{d_{\alpha}} R_{s_{k}}^{\alpha}(\tau) e^{j 2 \pi f_{o} \frac{(i-1)}{c} d \sin \theta_{k}} \quad \forall i=1,2, \ldots \ldots \ldots \ldots \ldots, M
$$

By collecting the correlation functions, given by (20), in a vector form

$$
\begin{gathered}
\mathbf{R}_{X}^{\alpha}(\tau)=\left[R_{x_{1}}^{\alpha}(\tau), \ldots \ldots \ldots \ldots, R_{x_{M}}^{\alpha}(\tau)\right]^{T}, \mathbf{R}_{X}^{\alpha}(\tau) \text { can be re-expressed as: } \\
\mathbf{R}_{X}^{\alpha}(\tau)=\mathbf{A}(\alpha) \mathbf{R}_{S}^{\alpha}(\tau)
\end{gathered}
$$

where $\mathbf{R}_{S}^{\alpha}(\tau)=\left[R_{S 1}^{\alpha}(\tau), R_{S 2}^{\alpha}(\tau), \ldots \ldots \ldots \ldots \ldots \ldots . . ., R_{S_{d \alpha}}^{\alpha}(\tau)\right]^{T}, \mathbf{A}(\alpha)$ is the array steering matrix which is represented as:

$$
\mathbf{A}(\alpha)=\left[\mathbf{a}_{1}(\alpha), \ldots \ldots \ldots \ldots, \mathbf{a}_{d_{\alpha}}(\alpha)\right]
$$

and $\mathbf{a}_{k}(\alpha)$ is the $k^{\text {th }}$ source steering vector, which is given by

$$
\mathbf{a}_{k}(\alpha)=\left[1, e^{j 2 \pi \alpha \frac{(i-1)}{c} d \sin \theta_{k}}, \ldots \ldots \ldots \ldots . . ., e^{j 2 \pi \alpha \frac{(i-1)}{c}(M-1) d \sin \theta_{k}}\right]^{T}
$$

Since $\mathbf{R}_{X}^{\alpha}(\tau)$ is independent of the intercepted signal center frequency, $f_{c}$, Therefore, whether the original data, which is collected at each sensor individually, is narrowband or wideband, the cyclic correlation of data exactly obeys the narrowband data model ,given by (10) with cyclic frequency $\alpha$ as a center frequency. Thus, it is possible to estimate the sources DOAs by applying conventional narrow band algorithms on $\mathbf{R}_{X}^{\alpha}(\tau)$.

\section{III - Simulation work}

Subsection A presents simulation of test signals and system parameters Subsection B compares between the performance of conventional MUSIC algorithm and the proposed cyclic MUSIC algorithm to estimate DOAs of narrowband signals either they are cyclically correlated or cyclically uncorrelated. Subsection $\mathrm{C}$ studies the effect of bandwidth of the SOI 
on the DOA estimation accuracy of cyclic MUSIC algorithm in presence of wideband interference signals either they are cyclically correlated or cyclically uncorrelated with SOI. Also, a comparison with conventional MUSIC algorithm accuracy is discussed. Subsection D presents the angle resolution of the cyclic MUSIC algorithm to distinguish between two cyclically correlated signals.

\section{A - Simulation of test signals and system parameters:}

According to the specification of the intercept receiver, the signal is down converted to IF frequency, $f_{I F}$, of $150 \mathrm{KHz}$ with sampling rate, $f_{s}$, of $1200 \mathrm{KHz}$. The modulating digital symbol sequence duration is chosen to be $1.707 \mathrm{msec}$ (equivalent to $\mathrm{N}=2048$ samples). The simulated signals are chosen to be real, which can be expressed as:

$$
S_{j}(i)=A_{j} \operatorname{COS}\left(\frac{2 \pi f_{I F} i}{f_{s}}+\Psi_{j}\right) ; \forall 1 \leq i \leq N_{b} \text { and } j=0,1,2, \ldots . ., K-1 ; K=2,4,8, . .
$$

where $K$ is the number of signal states that must be a modulo2 number and $N_{b}$ is the number of samples per symbol duration, which is equivalent to the ratio between the sampling

frequency, $f_{s}$, and the symbol rate, ${ }^{r}$. Also, in simulation of wideband signals, the measure of the bandwidth of the incident signal to be narrow or wide is defined as the ratio of the bandwidth of the complex base band signal and its carrier frequency [4, 7]. So, the simulated signal is called a wideband signal if this ratio is larger than $0.2[4,7]$. The simulated signals are assumed to have the same carrier frequency and an equal power which is $10 \mathrm{~dB}$ relative to the background white Gaussian noise and $\mathrm{SIR}=-3 \mathrm{~dB}$. Moreover, In cyclic MUSIC algorithm, it is assumed that the cyclic frequency of SOI and the optimal lag time, at which the source cyclic correlation $R_{s}^{\alpha}(\tau)$ achieves its maximum, are known or estimated $[4,7,8]$. The employed array is 11-element non-uniform linear array with the following inter-element

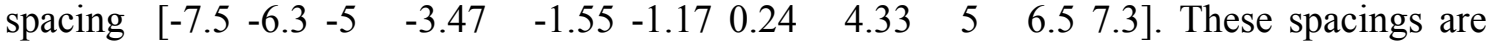
calculated relative to the wave length of SOI carrier frequency. The complete system is simulated using MATLAB software Ver.6.3 [9].

\section{B - Performance evaluation with narrow band signals}

\section{B.1- Cyclically uncorrelated signals environment}

The simulated signals are generated with the same parameters that are listed in subsection A. The desired signal is chosen to be 8-PSK that has $\frac{f_{s}}{60}$ bit rate, $f_{c}$ carrier frequency, and $0^{\circ}$ DOA. The cyclically uncorrelated interferer signals are 8-ASK and 8-QAM that have $\left\{\frac{f_{s}}{50}, \frac{f_{s}}{40}\right\}$ bit rates, $\left[f_{c}+20 \mathrm{KHz}, f_{c}-10 \mathrm{KHz}\right]$ carrier frequencies, and $\left[-40^{\circ}, 40^{\circ}\right]$ DOAs respectively. The three signal sources are assumed to have equal power which is $10 \mathrm{~dB}$ relative to the background white Gaussian noise and $\mathrm{SIR}=-3 \mathrm{~dB}$. It is clear that the 
interference signals are cyclically uncorrelated with SOI because they have different bit rates and different carrier frequencies. Consequently, they have different cyclic frequencies.

\section{B.1.1 - Performance of Conventional subspace algorithm}

DOA estimation layer searches for DOAs of the incident signals by using conventional MUSIC algorithm. Fig.1. shows the conventional MUSIC spatial spectrum where three peaks appear in the graph. After spectrum search of the peaks, the estimated values are $\left[-40^{\circ}, 0^{\circ}, 40^{\circ}\right]$ which are being the same DOAs of the simulated signals.

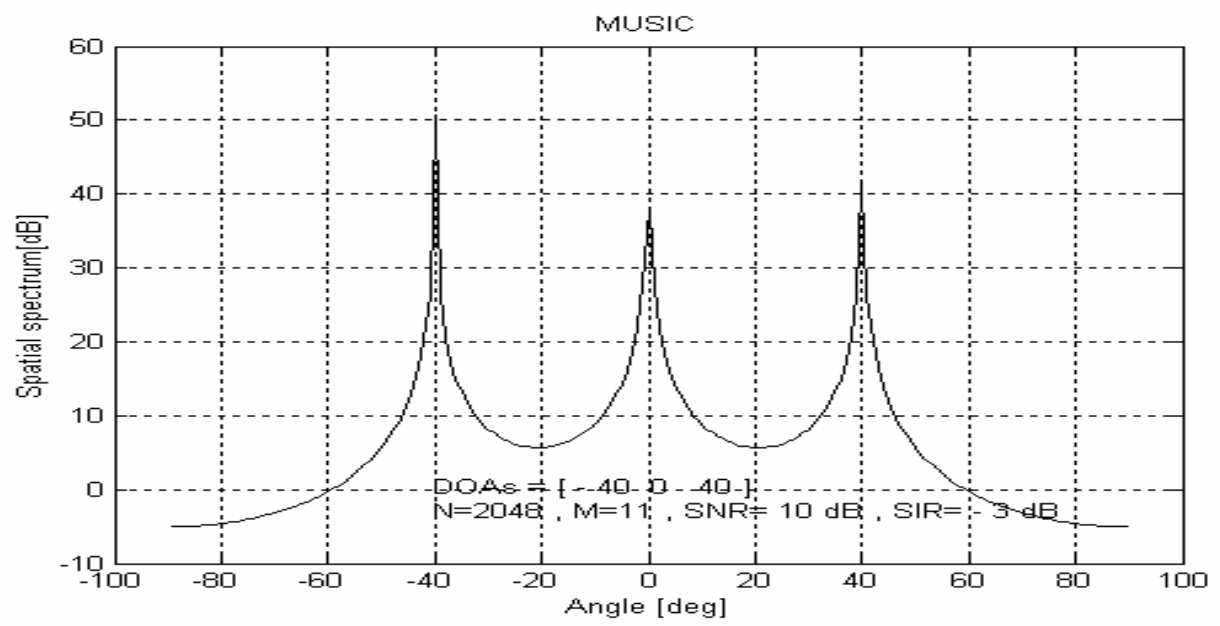

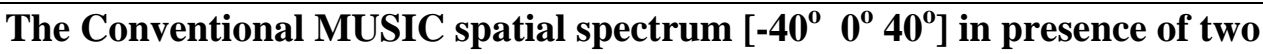

Fig.1. cyclically uncorrelated signals with SOI.

\section{B.1.2 - Performance of Cyclic subspace algorithm}

In this subsection, the cyclic MUSIC algorithm is used for DOAs estimation of the incident cyclically uncorrelated signals. Fig.2. shows the cyclic MUSIC spatial spectrum where only one peak appears in the graph. After spectrum search of the peaks, the estimated value, which is the DOA of SOI is $0^{\circ}$. Since the two interference signals are cyclically uncorrelated with SOI, their cyclic correlations are zero and are not appear in the spatial cyclic spectrum. 


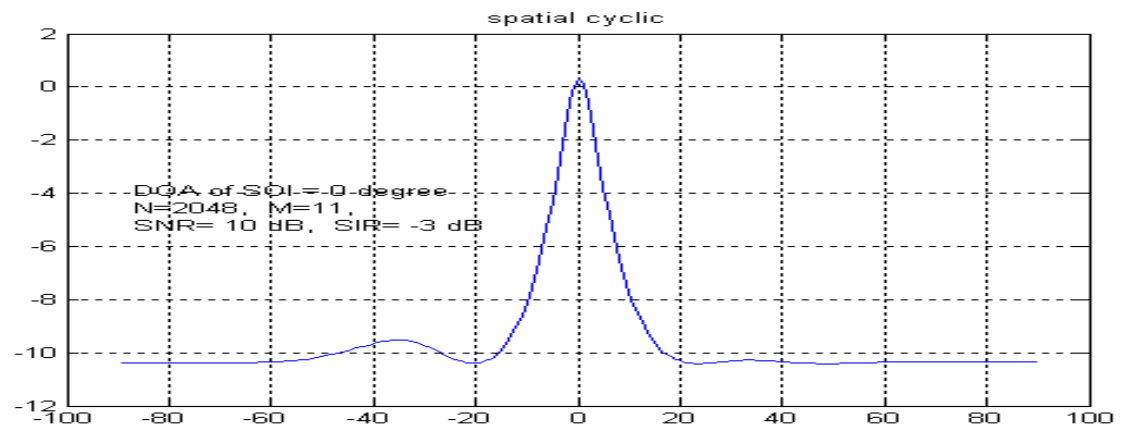

\section{The cyclic MUSIC spatial spectrum $\left[\begin{array}{lll}-40^{\circ} & 0^{\circ} & 40^{\circ}\end{array}\right]$ in presence of two cyclically $\quad$ Fig.2. uncorrelated signals with SOI.}

\section{B.2- Cyclically correlated signals environment}

The simulated signals are generated with the same parameters that are listed in subsection A. The array is illuminated by three signals. The first one is the desired signal which is chosen to $f_{s}$

be 8 -PSK that has 60 bit rate and $-40^{\circ}$ DOA. The second one is the cyclic correlated interference signal, which is a 8-PSK signal with the same bit rate and carrier frequency and has $0^{\circ}$ DOA. The third one is another interference signal which is a 8-QAM that has $\frac{f_{s}}{40}$ bit rate and $40^{\circ}$ DOA. Note that the 8-QAM signal is cyclically uncorrelated with the other two 8-PSK signals. While the two 8-PSK signals are cyclically correlated because they have the same carrier signal and bit rates. Consequently, they have the same cyclic frequencies.

\section{B.2.1 - Conventional subspace algorithm}

The results are shown in Fig.3. After spectrum search of the peaks, only one dominant peak appears that corresponds to DOA of the cyclically uncorrelated signal at $40^{\circ}$. Thus, conventional MUSIC algorithm fails to detect DOA of SOI in presence of cyclically correlated interference signals. 


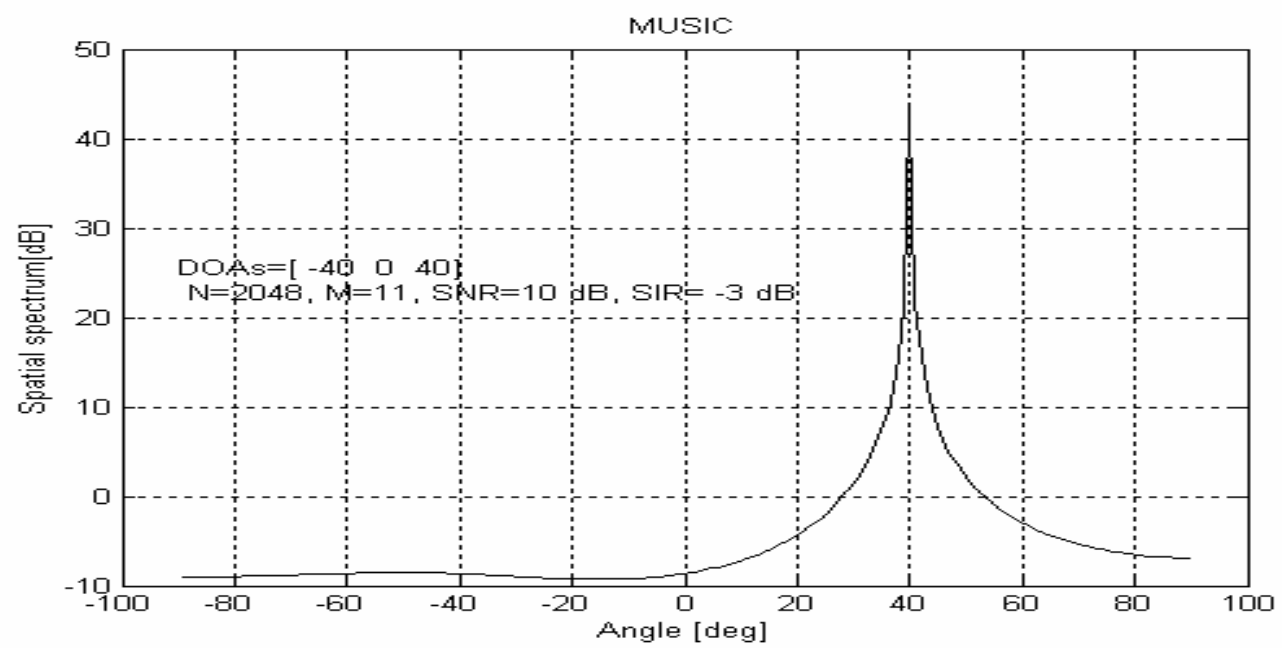

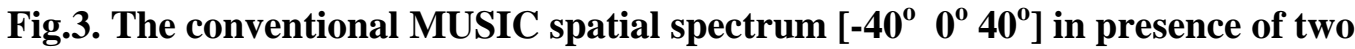
cyclically correlated signals and one cyclically uncorrelated signal

\section{B.2.2 - Cyclic subspace algorithm}

The results are shown in Fig.4. After spectrum search of the peaks, the estimated values are $\left[0^{\circ},-40^{\circ}\right]$ which are the DOAs of the simulated cyclic correlated signals whereas the cyclic uncorrelated interference signal does not appear in the spatial cyclic spectrum because its cyclic correlation is zero.

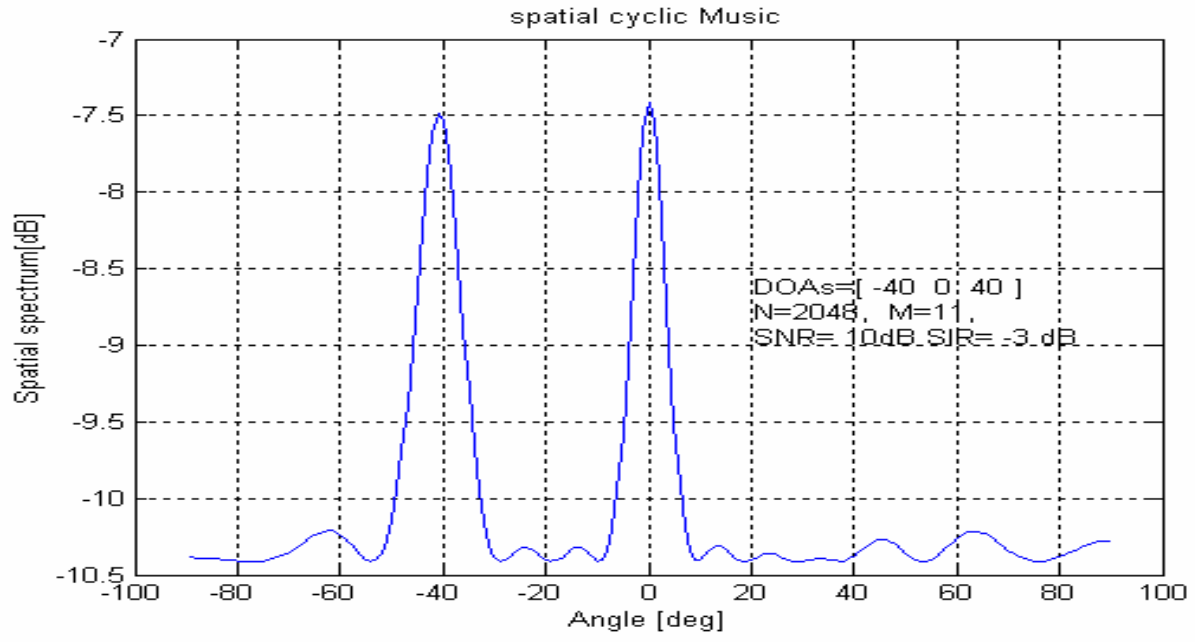

The cyclic MUSIC spatial spectrum $\left[\begin{array}{ccc}-40^{\circ} & 0^{\circ} & 40^{\circ}\end{array}\right]$ in presence of two cyclically correlated signals and one cyclically uncorrelated signal

Fig.4. 


\section{C - Effect of the band width on DOA estimation accuracy}

Here, the cyclic MUSIC algorithm is used to study the effect of the bandwidth on its DOA estimation accuracy in conjunction with the conventional MUSIC one. At first, the desired signal is chosen to be 8-QAM with $-40^{\circ}$ DOA and $\frac{f_{s}}{15}$ bit rate. The interference signal is $\frac{f_{s}}{20}$

chosen to be 8 -PSK that has $\overline{20}$ bit rate and $0^{\circ}$ DOA. Consequently, the two signals are cyclically uncorrelated. The bandwidth of the signals is frequently changed in the range $0.05 f_{c}$ to $0.5 f_{c}$ by step $0.05 f_{c}$ and each time the DOAs are measured. Note that this range of frequencies covers the bandwidth of narrowband and wideband signals. The estimation accuracy of DOA is defined as the absolute of the estimated DOA deviation from the correct angle. In Fig.5., the correct desired DOA has red color while the correct interference DOA has blue color. Fig.5.a. shows that the accuracy gets worse as the bandwidth increases in case of using conventional MUSIC algorithm because it is based on narrowband assumption. Fig.5.b. shows that the accuracy has small variations as the bandwidth of SOI increases. Secondly, the interference signal is chosen to be of the same type of SOI with the same bit rate, carrier frequency, and $40^{\circ}$ DOA. Consequently, the two incident signals have the same cyclic frequencies. Thus, they are cyclically correlated. Fig.5.c. illustrates that cyclic MUSIC algorithm accuracy is stable around the true DOAs. Thus, cyclic MUSIC algorithm has stable accuracy with the increment of the incident signals bandwidths either they are cyclically correlated or cyclically uncorrelated.

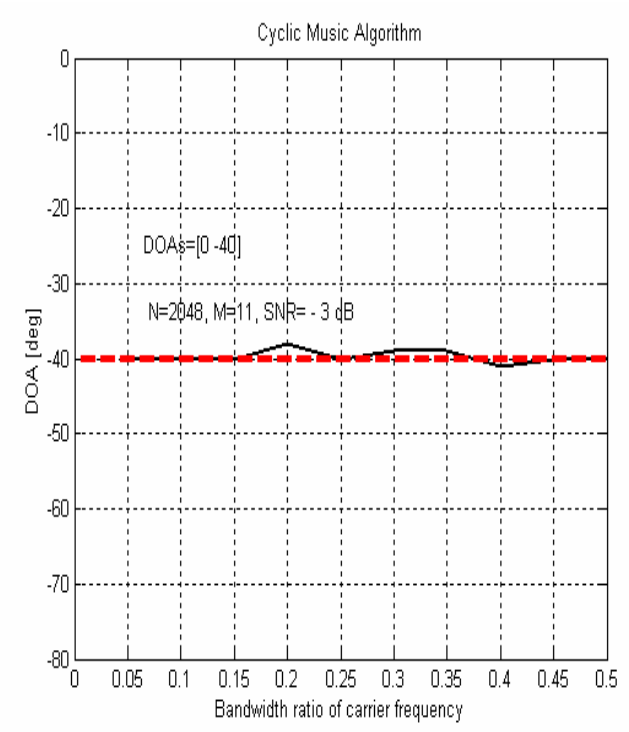

(b ) cyclic MUSIC algorithm (non-coherent case)

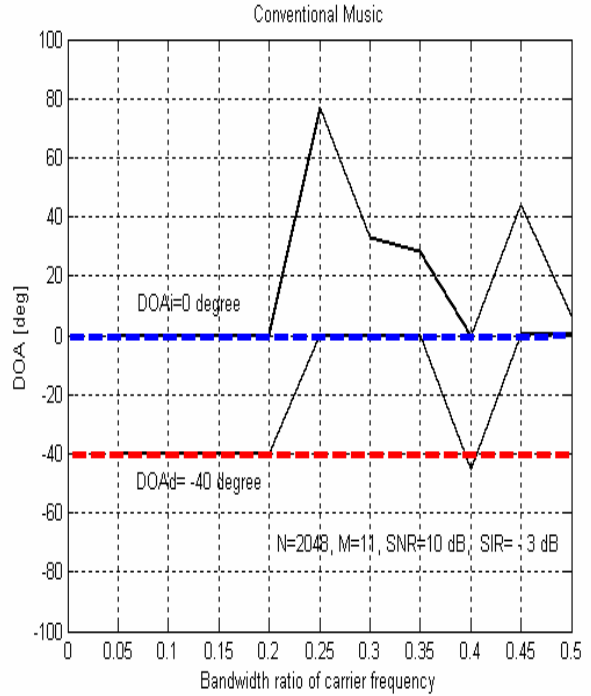

(a) conventional MUSIC algorithm 


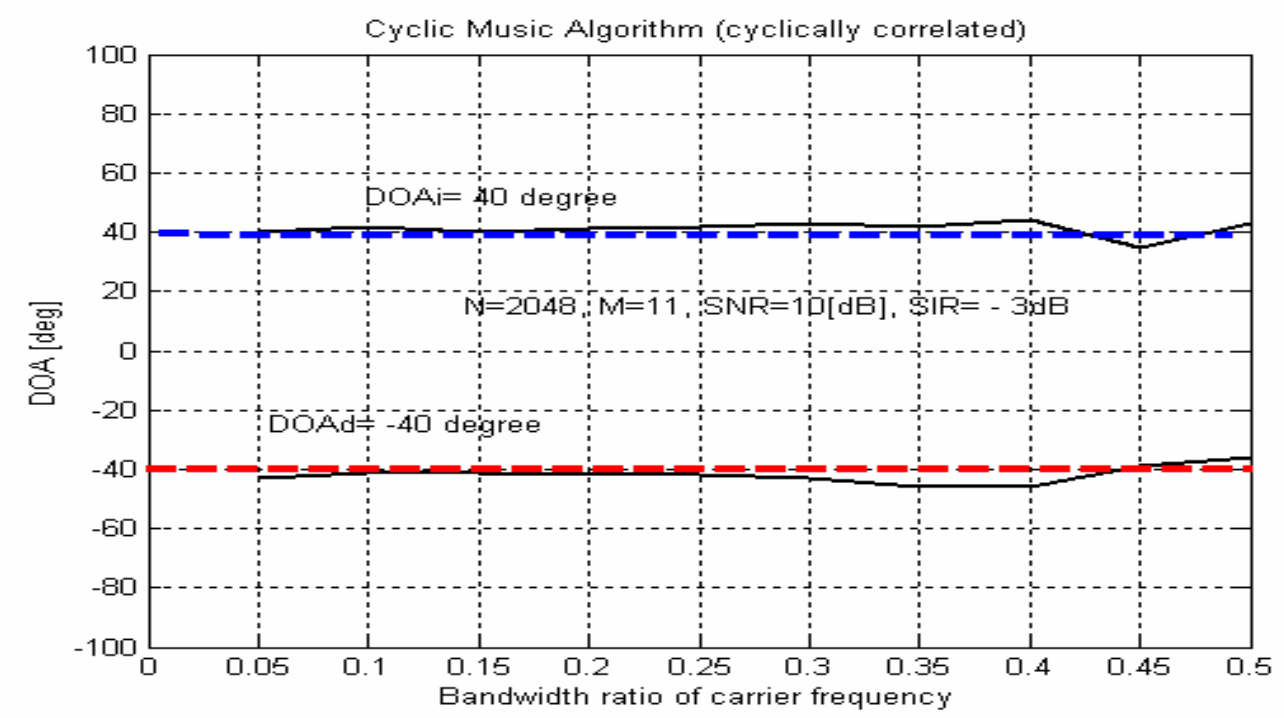

(c) cyclic MUSIC algorithm ( coherent case )

The accuracy as SOI bandwidth increases $\left(0.05 f_{c}: 0.5 f_{c}\right.$

Fig.5.

\section{D - Resolution}

In general, the resolution can be defined as the minimum difference in a measurable value of certain property of two signals that have the same other properties except the tested one such that both signals can be discriminated. In this subsection, the evaluated resolution is in angle of arrival. The same assumptions of the antenna array parameters in subsection A are used except that the array is illuminated by two BPSK signals with equal power, which is $10 \mathrm{~dB}$ relative to the background white Gaussian noise. In order to measure the resolution in angle of arrival, it is assumed that both signals have the same carrier frequency and the same bit rate. Thus, the two signals have the same cyclic frequency. Consequently, they are cyclically correlated and can not be distinguished by using conventional MUSIC algorithm as indicated in subsection B.2.1. Fig.6.a. shows the spatial spectrum of cyclic MUSIC algorithm in order to estimate DOAs of two BPSK signals that are simulated with $\left[\begin{array}{ll}0^{\circ} & 6.4^{\circ}\end{array}\right]$ DOAs. The estimated DOAs are $\left[\begin{array}{ll}0^{\circ} & 6^{\circ}\end{array}\right]$. SinFig.5.6.b. shows that cyclic MUSIC algorithm fails to estimate DOAs of two BPSK signals that are simulated with $\left[\begin{array}{lll}0^{\circ} & 6.3^{\circ}\end{array}\right]$ DOAs. The spatial spectrum shows only one peak that corresponds to $5^{\circ}$. 


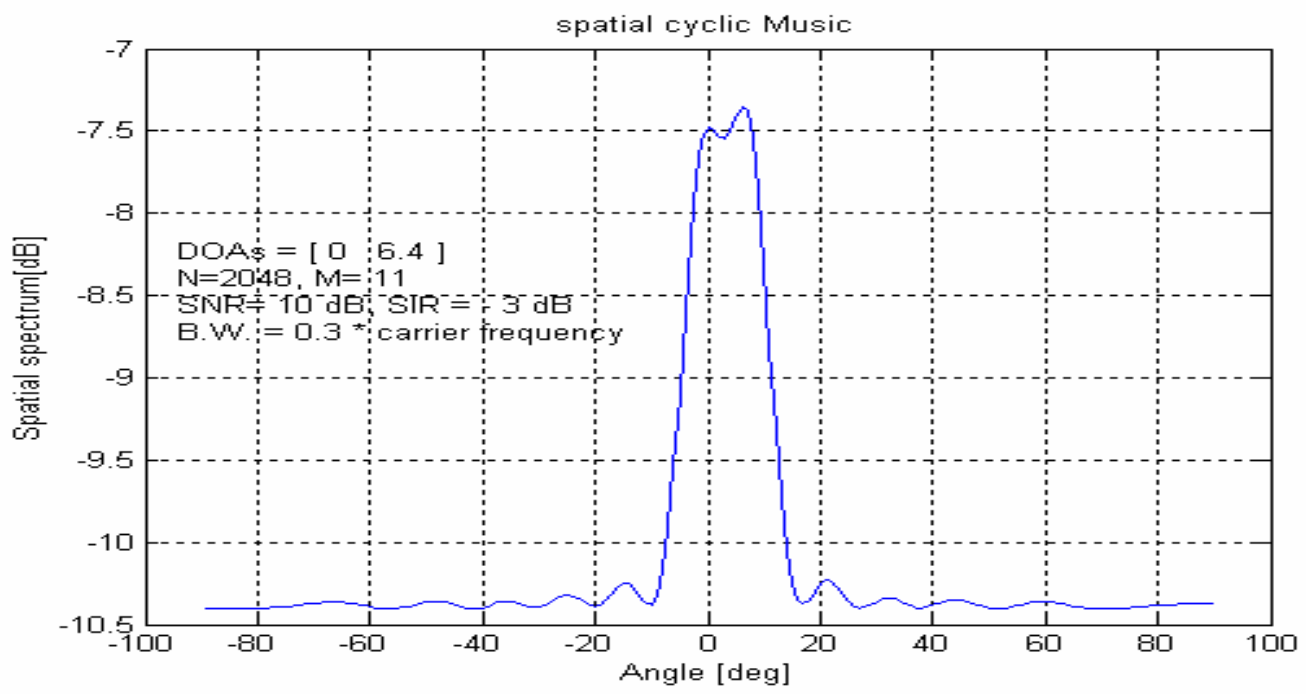

(a)

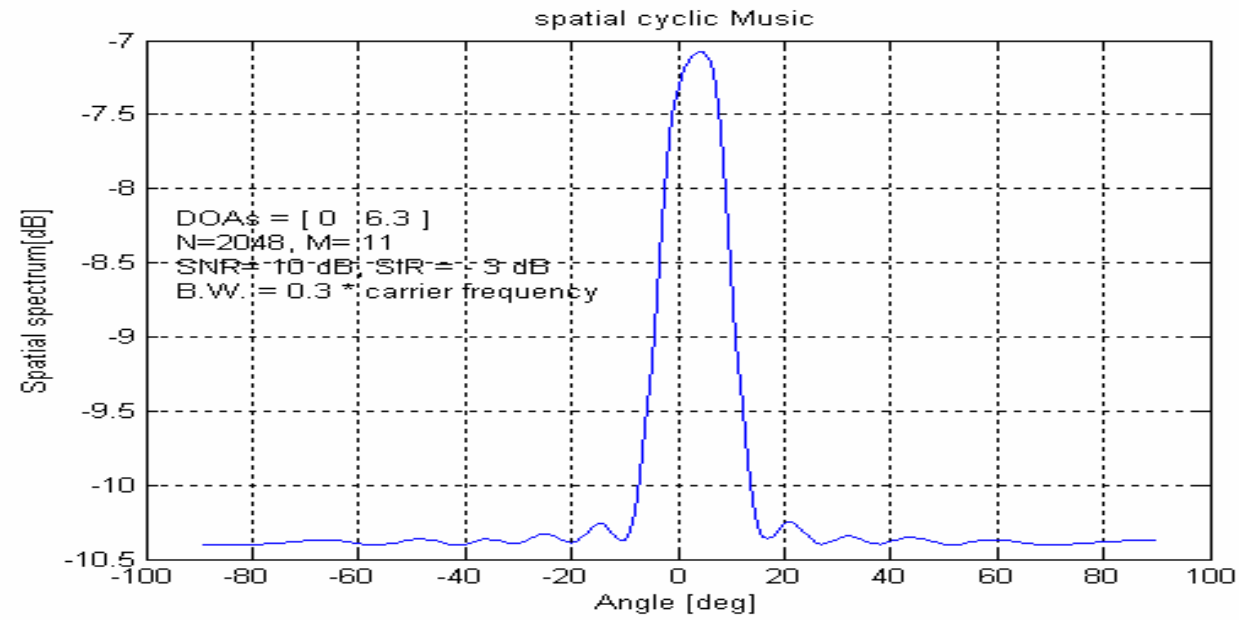

(b)

Angle resolution of the cyclic MUSIC DOA algorithm in presence of two cyclically correlated signals when the simulated DOAs are:

(a)- $\left[\begin{array}{ll}0^{\circ} & 6.4^{0}\end{array}\right]$, (b)- $\left[\begin{array}{ll}0^{\circ} & 6.3^{\circ}\end{array}\right]$

Fig.5.6.

\section{IV- Conclusions}

The performance measures and comparisons has been carried out for conventional MUSIC and the proposed cyclic MUSIC algorithms using computer simulations. It has been found that:

Conventional MUSIC algorithm has been succeeded to estimate DOAs of cyclically uncorrelated narrowband signals but it has been failed to estimate DOAs of cyclically correlated narrowband signals. Conventional MUSIC algorithm has been failed to estimate DOAs of wideband cyclically uncorrelated signals. DOA estimation accuracy by using conventional MUSIC algorithm gets worse as the bandwidth of the SOI increases. The 
proposed cyclic MUSIC algorithm has been succeeded to estimate DOAs of both narrowband and wideband signals either they are cyclically correlated or cyclically uncorrelated. DOA estimation accuracy by using the proposed cyclic MUSIC algorithm is stable with the increment of the SOI bandwidth either in presence of cyclically correlated or cyclically uncorrelated signals. The AAA system is able to distinguish between two signals in angle.

\section{Appendix ( A ):}

If $\mathbf{x}($.$) is cyclostationary process with self cyclic correlation function \mathbf{R}_{x}^{\alpha}(\tau)$, and $y(t)$ is a delayed version of $x(t)$ where $y(t)=\mathbf{x}(t+T)$, then [33]

$$
\mathbf{R}_{y}^{\alpha}(\tau)=\mathbf{R}_{x}^{\alpha}(\tau) e^{+j 2 \pi \alpha T}
$$

proof

$$
\begin{aligned}
\mathbf{R}_{y}^{\alpha}(\tau) & =\prec y\left(t+\frac{\tau}{2}\right) y^{*}\left(t-\frac{\tau}{2}\right) e^{-j 2 \pi \alpha t} \succ \\
& =\prec x\left(t+T+\frac{\tau}{2}\right) x^{*}\left(t+T-\frac{\tau}{2}\right) e^{-j 2 \pi \alpha t} \succ \\
& =\prec x\left(t^{\prime}+\frac{\tau}{2}\right) x^{*}\left(t^{\prime}-\frac{\tau}{2}\right) e^{-j 2 \pi \alpha t} \succ
\end{aligned}
$$

By denoting $t^{\prime}=t+T$, the $\mathbf{R}_{y}^{\alpha}(\tau)$ can be written as:

$$
\mathbf{R}_{y}^{\alpha}(\tau)=\mathbf{R}_{x}^{\alpha}(\tau) e^{-j 2 \pi \alpha T}
$$

It is clear that cyclic correlation function, $\mathbf{R}_{x}^{\alpha}(\tau)$, has important property that it is not a function of the time variable $t$. Also, the time delay $T$ is transformed into a phase shift.

\section{V-References}

[1] H. Krim, Mats Viberg," Two decades of array signal Processing research”, IEEE Signal Processing Magazine, pp.67-94, July 1996.

[2] W.A. Gardner, Cyclostationarity in Communications and Signal Processing, IEEE Press, NY, 1994.

[3] Qiang Wu and Kon Max Wong, "Blind Adaptive Beamforming for cyclostationary signals "IEEE Transactions on Signal Processing, Vol.44, No.11, November 1996.

[4] G.Xu and T. Kailath, "Direction of arrival Estimation via Exploitation of cyclostationarity-A combination of temporal and Spatial processing", IEEE Transactions on Signal Processing, juluy 1992.

[5] M. Yao, L. Jin, Q. Yin, "Selective Direction Finding for Cyclostationary Signals by Exploitation of New Array Configuration," ICASSP 1999.

[6] F. Ridder, R. Pintelon, J. Schoukens, and D. Gillikin,” Modified AIC and MDL Model Selection Criteria for Short Data Records", IEEE Trans. On Instrumentation and Measurement, Vol. 54, no. 1, February 2005.

[7] W.A. Gardner, Cyclostationarity in Communications and Signal Processing, IEEE Press, NY, 1994.

[8] S. Naidu, Prabhaker, "Sensor Array Signal Processing “ CRC Press, 2001. 
[9] Andrew Knigh, "Basics of matlab and beyond”, Champion Hall, Florida, 2000. 